\title{
A COMMON FIXED POINT THEOREM ON FUZZY METRIC SPACE
}

\author{
V. Srinivas*, B. V. B. Reddy, R. Umamaheshwar Rao \\ Department of Mathematics, Sreenidhi Institute of Science and Technology, Ghatkesar \\ R.R.Dist-501 301, Andhra Pradesh, India. \\ *Corresponding author: srinivasveladi@yahoo.co.in \\ Received 22 February, 2012; Revised 21 August, 2012
}

\begin{abstract}
The purpose of this paper is to prove a common fixed point theorem in a fuzzy metric space using the concept of weakly compatible mappings.
\end{abstract}

Keywords: Fixed point, self maps, weakly compatible mappings.

AMS (2000) Mathematics Classification: 54H25, 47H10

\section{INTRODUCTION}

The concept of the fuzzy metric spaces has been introduced by Zadeh [1], in the recent past many authors developed the theory of fuzzy sets and applications. Grabic [4] obtained the Banach contraction principle in fuzzy version. Recently George and Veeramani [3] have shown that every metric induces a fuzzy metric.

\section{Definitions and Preliminaries}

Definition 1.1: A binary operation $*:[0,1] \times[0,1] \rightarrow[0,1]$ is called continuous $t$-norm if $*$ satisfies the following conditions:

(i) $\quad *$ is commutative and associative

(ii) $*$ is continuous

(iii) $\mathrm{a}^{*} 1=\mathrm{a}$ for all $\mathrm{a} \in[0,1]$

(iv) $\quad a^{*} b \leq c^{*} d$ whenever $a \leq c$ and $b \leq d$ for all $a, b, c, d \in[0,1]$

Definition 1.2: A 3-tuple $(X, M, *)$ is said to be fuzzy metric space if $X$ is an arbitrary set,* is continuous $t-$ norm and $M$ is a fuzzy set on $X^{2} \times(0, \infty)$ satisfying the following conditions for all $\mathrm{x}, \mathrm{y}, \mathrm{z} \in \mathrm{X}, \mathrm{s}, \mathrm{t}>0$

$(\mathrm{FM}-1) \quad \mathrm{M}(\mathrm{x}, \mathrm{y}, 0)=0$

(FM-2) $M(x, y, t)=1$ for all $t>0$ if and only if $x=y$

$(\mathrm{FM}-3) \quad \mathrm{M}(\mathrm{x}, \mathrm{y}, \mathrm{t})=\mathrm{M}(\mathrm{y}, \mathrm{x}, \mathrm{t})$

$(\mathrm{FM}-4) \mathrm{M}(\mathrm{x}, \mathrm{y}, \mathrm{t}) * \mathrm{M}(\mathrm{y}, \mathrm{z}, \mathrm{s}) \leq \mathrm{M}(\mathrm{x}, \mathrm{z}, \mathrm{t}+\mathrm{s})$

$(\mathrm{FM}-5) \mathrm{M}(\mathrm{x}, \mathrm{y},):.[0, \infty) \rightarrow[0,1]$ is left continuous

The function $M(x, y, t)$ denote the degree of nearness between $x$ and $y$ with respect to ' $t$ '. We observe $M(x, y, t)=1$ when $x=y$ for all $t>0$ and $M(x, y, t)=0$ with $t=\infty$. Some of the properties of fuzzy metric space and examples are given in paper George and Veeramani.

Example 1.3 (Induced fuzzy metric space): Let $(X, d)$ be a metric space defined $a * b=\min \{a, b\}$ for all $x, y \in X$ and $t>0$, 


$$
M(x, y, t)=\frac{t}{t+d(x, y)}
$$

Then $\left(\mathrm{X}, \mathrm{M},{ }^{*}\right)$ is a fuzzy metric space. We call this fuzzy metric $M$ induced by metric $d$ the standard fuzzy metric. From the above example every metric induces a fuzzy metric but there exist no metric on X satisfying (a).

Definition 1.4: Let $\left(X, M,{ }^{*}\right)$ be a fuzzy metric space then a sequence $\left\langle x_{n}\right\rangle$ in $X$ is said to be convergent to a point $\mathrm{x} \in \mathrm{X}$, if $\lim _{n \rightarrow \infty} M\left(x_{n}, x, t\right)=1$ for all $t>0$.

Definition 1.5: A sequence $\left\langle\mathrm{x}_{\mathrm{n}}\right\rangle$ in $\mathrm{X}$ is called a Cauchy sequence if $\lim _{n \rightarrow \infty} M\left(x_{n+p}, x_{n}, t\right)=1$ for all $t>0$ and $p>0$.

Definition 1.6: A fuzzy metric space $\left(\mathrm{X}, \mathrm{M},{ }^{*}\right)$ is said to be complete if every Cauchy sequence is convergent to a point in $\mathrm{X}$.

Lemma 1.7 ([4]): For all $\mathrm{x}, \mathrm{y} \in \mathrm{X}, \mathrm{M}(\mathrm{x}, \mathrm{y},$.$) is non decreasing.$

Lemma 1.8 ([5]) let $(X, M, *)$ be a fuzzy metric space if there exists $k \in(0,1)$ such that $\mathrm{M}(\mathrm{x}, \mathrm{y}, \mathrm{kt}) \geq \mathrm{M}(\mathrm{x}, \mathrm{y}, \mathrm{t})$ then $\mathrm{x}=\mathrm{y}$.

Proposition 1.9 ([3]): In the fuzzy metric space $(X, M, *)$ if $a * a \geq a$ for all $a \in[0,1]$ then $a^{*} b=$ $\min \{a, b\}$

Definition 1.10: Two self maps $S$ and $T$ of a fuzzy metric space $\left(X, M,{ }^{*}\right)$ are said to be compatible mappings if $\lim _{n \rightarrow \infty} \mathrm{M}\left(\mathrm{STx}_{\mathrm{n}}, \mathrm{TSx}_{\mathrm{n}}, \mathrm{t}\right)=1$, whenever $\left\langle\mathrm{x}_{\mathrm{n}}\right\rangle$ is a sequence in $\mathrm{X}$ such that $\lim _{\mathbf{n} \rightarrow \infty} \mathrm{Sx}_{\mathrm{n}}=\lim _{\mathbf{n} \rightarrow \infty} \mathrm{Tx}_{\mathrm{n}}=\mathrm{z}$ for some $\mathrm{z} \in \mathrm{X}$.

Definition 1.11: Two self maps $S$ and $T$ of a fuzzy metric space $(X, M, *)$ are said to be weakly compatible if they commute at their coincidence point. i.e if $\mathrm{Su}=\mathrm{Tu}$ for some $\mathrm{u} \in \mathrm{X}$ then $\mathrm{STu}=\mathrm{TSu}$.

It is clear that every compatible pair is weakly compatible but its converse need not be true.

To know the relation among commutatively, compatibility and weakly compatibility refer some of the research papers like Jungck[7], Pant[11], Srinivas and others[12].

2.1 Theorem: Let $\mathrm{A}, \mathrm{B}, \mathrm{S}$ and $\mathrm{T}$ are self maps of a complete Fuzzy metric space $\left(\mathrm{X}, \mathrm{M},{ }^{*}\right)$ satisfying the conditions

$$
\mathrm{A}(\mathrm{X}) \subseteq \mathrm{T}(\mathrm{X}) \text { and } \mathrm{B}(\mathrm{X}) \subseteq \mathrm{S}(\mathrm{X})
$$

The pairs $(\mathrm{A}, \mathrm{S})$ and $(\mathrm{B}, \mathrm{T})$ are weakly compatible

$$
\begin{gathered}
{[M(A x, B y, k t)]^{2} * M(A x, B y, k t) M(T y, S x, k t) \geq\left\{k_{1}[M(B y, S x, 2 k t) * M(A x, T y, 2 k t)]\right.} \\
\left.+k_{2}[M(A x, S x, k t) * M(B y, T y, k t)]\right\} M(T y, S x, t)
\end{gathered}
$$

Where for all $\mathrm{x}, \mathrm{y}$ in $\mathrm{X}$ and $\mathrm{k}_{1}, \mathrm{k}_{2} \geq 0, \mathrm{k}_{1}+\mathrm{k}_{2} \geq 1$

Then $\mathrm{A}, \mathrm{B}, \mathrm{S}$ and $\mathrm{T}$ have a unique common fixed point $\mathrm{z}$ in $\mathrm{X}$. 
(2.1.4) Lemma: Let $A, B, S$ and $T$ be self mappings from a complete metric space $(X, d)$ into itself satisfying the conditions (2.1.1)and (2.1.3)Then the sequence $\left\{\mathrm{y}_{\mathrm{n}}\right\}$ defined by $\mathrm{y}_{2 \mathrm{n}}=\mathrm{Ax}_{2 \mathrm{n}}=\mathrm{Tx}_{2 \mathrm{n}+1}$ and $\mathrm{y}_{2 \mathrm{n}+1}=\mathrm{Bx}_{2 \mathrm{n}+1}=\mathrm{Sx}_{2 \mathrm{n}+2}$ for $\mathrm{n} \geq 0$ relative to four self maps is a cauchy sequence in $\mathrm{X}$.

Proof: From the conditions (2.1.1) (2.1.3) and from the definition of associated sequence we have

Let $\mathrm{x}_{0}$ be any arbitrary point of $\mathrm{X}, \mathrm{A}(\mathrm{X}) \subseteq \mathrm{T}(\mathrm{X})$ and $\mathrm{B}(\mathrm{X}) \subseteq \mathrm{S}(\mathrm{X})$ there exists $\mathrm{x}_{1}, \mathrm{x}_{2} \in \mathrm{X}$ such that $\mathrm{Ax}_{0}=\mathrm{Tx}_{1}$ and $\mathrm{Bx}_{1}=\mathrm{Sx}_{2}$.

Inductively we construct a sequence $\left\langle\mathrm{x}_{\mathrm{n}}\right\rangle$ and $\left\langle\mathrm{y}_{\mathrm{n}}\right\rangle$ in $\mathrm{X}$ such that $\mathrm{y}_{2 \mathrm{n}}=\mathrm{Ax}_{2 \mathrm{n}}=\mathrm{Tx}_{2 \mathrm{n}+1}$ and $\mathrm{y}_{2 \mathrm{n}+1}=\mathrm{Bx}_{2 \mathrm{n}+1}=\mathrm{Sx}_{2 \mathrm{n}+2}$ for $\mathrm{n} \geq 0$.

By taking $\mathrm{x}=\mathrm{x}_{2 \mathrm{n}} \quad \mathrm{y}_{=} \mathrm{x}_{2 \mathrm{n}+1}$

$$
\begin{aligned}
& {\left[M\left(A x_{2 n}, B x_{2 n+1}, k t\right)\right]^{2} * M\left(A x_{2 n}, B x_{2 n+1}, k t\right) M\left(T x_{2 n+1}, S x_{2 n}, k t\right) \geq\left\{k_{1}\left[M\left(B x_{2 n+1}, S x_{2 n}, 2 k t\right) * M\left(A v, T x_{2 n+1} 2 k t\right)\right]\right.} \\
& \left.+k_{2}\left[M\left(A x_{2 n}, S x_{2 n}, k t\right) * M\left(B x_{2 n+1}, T x_{2 n+1}, k t\right)\right]\right\} M\left(T x_{2 n+1}, S x_{2 n}, t\right) \\
& {\left[M\left(y_{2 n}, y_{2 n+1}, k t\right)\right]^{2} * M\left(y_{2 n}, y_{2 n+1}, k t\right) M\left(y_{2 n}, y_{2 n-1}, k t\right) \geq\left\{k_{1}\left[M\left(y_{2 n+1}, y_{2 n-1}, 2 k t\right)\right) * M\left(y_{2 n}, y_{2 n}, 2 k t\right)\right]} \\
& \left.+k_{2}\left[M\left(y_{2 n}, y_{2 n-1}, k t\right) * M\left(y_{2 n+1}, y_{2 n}, k t\right)\right]\right\} M\left(y_{2 n}, y_{2 n-1}, t\right) \\
& {\left[M\left(y_{2 n}, y_{2 n+1}, k t\right)\right]\left\{M\left(y_{2 n}, y_{2 n+1}, k t\right) * M\left(y_{2 n}, y_{2 n-1}, k t\right)\right\} \geq\left\{k_{1}\left[M\left(y_{2 n+1}, y_{2 n-1}, 2 k t\right)\right]\right.} \\
& \left.+k_{2}\left[M\left(y_{2 n}, y_{2 n-1}, k t\right) * M\left(y_{2 n+1}, y_{2 n}, k t\right)\right]\right\} M\left(y_{2 n}, y_{2 n-1}, t\right) \\
& {\left[M\left(y_{2 n}, y_{2 n+1}, k t\right)\right]\left\{M\left(y_{2 n+1}, y_{2 n-1}, 2 k t\right)\right\} \geq\left\{k_{1}\left[M\left(y_{2 n+1}, y_{2 n-1}, 2 k t\right)\right]+k_{2}\left[M\left(y_{2 n-1}, y_{2 n+1}, 2 k t\right)\right]\right\} M\left(y_{2 n}, y_{2 n-1}, t\right)} \\
& {\left[M\left(y_{2 n}, y_{2 n+1}, k t\right)\right]\left[M\left(y_{2 n+1}, y_{2 n-1}, 2 k t\right)\right] \geq\left\{k_{1}\left[M\left(y_{2 n+1}, y_{2 n-1}, 2 k t\right)\right]+k_{2}\left[M\left(y_{2 n-1}, y_{2 n+1}, 2 k t\right)\right]\right\} M\left(y_{2 n}, y_{2 n-1}, t\right)} \\
& {\left[M\left(y_{2 n}, y_{2 n+1}, k t\right)\right]\left[M\left(y_{2 n+1}, y_{2 n-1}, 2 k t\right)\right] \geq\left\{k_{1}+k_{2}\right\}\left[M\left(y_{2 n+1}, y_{2 n-1}, 2 k t\right)\right] M\left(y_{2 n}, y_{2 n-1}, t\right)} \\
& {\left[M\left(y_{2 n}, y_{2 n+1}, k t\right)\right] \geq\left\{k_{1}+k_{2}\right\} M\left(y_{2 n-1}, y_{2 n}, t\right)} \\
& {\left[M\left(y_{2 n}, y_{2 n+1}, k t\right)\right] \geq M\left(y_{2 n-1}, y_{2 n}, t\right)} \\
& {\left[M\left(y_{n}, y_{n+1}, k t\right)\right] \geq M\left(y_{n-1}, y_{n}, t\right)} \\
& M\left(y_{n}, y_{n+1}, t\right) \geq M\left(y_{n-1}, y_{n}, t / k\right) \geq M\left(y_{n-1}, y_{n}, t / k^{2}\right) \geq M\left(y_{n-1}, y_{n}, t / k^{3}\right) \ldots \ldots . . M\left(y_{n-1}, y_{n}, t / k^{n}\right)
\end{aligned}
$$

This implies $\mathrm{M}\left(\mathrm{y}_{\mathrm{n}}, \mathrm{y}_{\mathrm{n}+1}, \mathrm{t}\right) \rightarrow 1$ as $\mathrm{n} \rightarrow \infty$

For each $\varepsilon>0$ and $\mathrm{t}>0$ we can choose $\mathrm{n}_{0} \in \mathrm{N}$ such that $\mathrm{M}\left(\mathrm{y}_{\mathrm{n}}, \mathrm{y}_{\mathrm{n}+1}, \mathrm{t}\right)>1-\varepsilon$

For $\mathrm{m}, \mathrm{n} \in \mathrm{N}$ suppose $\mathrm{m} \geq \mathrm{n}$

$$
\begin{aligned}
M\left(y_{n}, y_{m}, t\right) \geq & {\left[M\left(y_{n}, y_{n+1}, t / m-n\right) * M\left(y_{n+1}, y_{n+2}, t / m-n\right) * \ldots * M\left(y_{m-1}, y_{m}, t / m-n\right)\right] } \\
& \geq(1-\varepsilon) *(1-\varepsilon)^{*} \ldots . .(1-\varepsilon) \\
& \geq(1-\varepsilon)
\end{aligned}
$$

This shows that the sequence $\left\{y_{n}\right\}$ is a cauchy sequence in $X$ and, it converges to a limit, say $\mathrm{z} \in \mathrm{X}$. 


\section{Proof of main Theorem:}

$\mathrm{Ax}_{2 \mathrm{n}}, \rightarrow \mathrm{Z} \quad \mathrm{Tx}_{2 \mathrm{n}+1} \rightarrow \mathrm{Z} \quad, \quad \mathrm{Bx}_{2 \mathrm{n}+1} \rightarrow \mathrm{Z}, \quad \mathrm{Tx}_{2 \mathrm{n}+1} \rightarrow \mathrm{Z}$

Since the condition $\mathrm{A}(\mathrm{X}) \subseteq \mathrm{T}(\mathrm{X})$ implies there exists $\mathrm{u} \in \mathrm{X}$ such that $\mathrm{Tu}=\mathrm{Z}$

From the condition $\mathrm{B}(\mathrm{X}) \subseteq \mathrm{S}(\mathrm{X})$ implies there exists $\mathrm{v} \in \mathrm{X}$ such that $\mathrm{Sv}=\mathrm{Z}$

To prove $\mathrm{Av}=\mathrm{z}$

Put $\mathrm{x}=\mathrm{v}, \mathrm{y}=\mathrm{x}_{2 \mathrm{n}+1}$.

$$
\begin{aligned}
& {\left[M\left(A v, B x_{2 n+1}, k t\right)\right]^{2} * M\left(A v, B x_{2 n+1}, k t\right) M\left(T x_{2 n+1}, S v, k t\right) \geq\left\{k_{1}\left[M\left(B x_{2 n+1}, S v, 2 k t\right) * M\left(A v, T, x_{2 n+1} 2 k t\right)\right]\right.} \\
& \left.+k_{2}\left[M(A v, S v, k t) * M\left(B x_{2 n+1}, T x_{2 n+1}, k t\right)\right]\right\} M\left(T x_{2 n+1}, S v, t\right) \\
& {[M(A v, z, k t)]^{2} * M(A v, z, k t) M(z, z, k t) \geq\left\{k_{1}[M(z, z, 2 k t) * M(A v, z, 2 k t)]\right.} \\
& \left.+k_{2}[M(A v, z, k t) * M(z, z, k t)]\right\} M(z, z, t) \\
& {[M(A v, z, k t)]^{2} * M(A v, z, k t) \geq\left\{k_{1}[M(A v, z, 2 k t)]\right.} \\
& \left.+k_{2}[M(A v, z, k t)]\right\} M(z, z, t) \\
& \left\{[M(A v, z, k t)][M(A v, z, k t)]^{*} M(A v, z, k t)\right\} \geq\left\{k_{1}[M(A v, z, 2 k t)]\right. \\
& \left.+k_{2}[M(A v, z, k t)]\right\} M(z, z, t) \\
& \{[M(A v, z, k t)][M(A v, z, k t)]\} \geq\left\{k_{1}[M(A v, z, k t)]\right. \\
& \left.+k_{2}[M(A v, z, k t)]\right\} M(z, z, t) \\
& {[M(A v, z, k t)]^{2} \geq\left\{k_{1}+k_{2}\right\}[M(A v, z, k t)]} \\
& {[M(A v, z, k t)] \geq\left\{k_{1}+k_{2}\right\}} \\
& {[M(A v, z, k t)] \geq 1} \\
& \therefore A v=z
\end{aligned}
$$

Since, $\left(k_{2}+k_{1}\right) \geq 1$, where $k_{1}, k_{2} \geq 0$ get $\mathrm{Av}=\mathrm{z}$. Thus $\mathrm{Av}=\mathrm{Sv}=\mathrm{z}$.

Since the pair $(\mathrm{A}, \mathrm{S})$ is weakly compatible and $\mathrm{Av}=\mathrm{Sv}=\mathrm{z}$ implies $\mathrm{ASv}=\mathrm{SAv}$ or $\mathrm{Az}=\mathrm{Sz}$.

To prove $\mathrm{Bu}=\mathrm{z}, \quad$ Let $\mathrm{x}=\mathrm{x}_{2 \mathrm{n}}, \mathrm{y}=\mathrm{u}$

$$
\begin{aligned}
& \begin{array}{l}
{\left[M\left(A x_{2 n}, B u, k t\right)\right]^{2} * M\left(A x_{2 n}, B u, k t\right) M\left(T u, S x_{2 n}, k t\right) \geq\left\{k_{1}\left[M\left(B u, S x_{2 n}, 2 k t\right) * M\left(A x_{2 n}, T u, 2 k t\right)\right]\right.} \\
\left.\quad+k_{2}\left[M\left(A x_{2 n}, S x_{2 n}, k t\right) * M(B u, T u, k t)\right]\right\} M\left(T u, S x_{2 n}, t\right)
\end{array} \\
& {[M(z, B u, k t)]^{2} * M(z, B u, k t) M(z, z, k t) \geq\left\{k_{1}[M(B u, z, 2 k t) * M(z, z, 2 k t)]+k_{2}[M(z, z, k t) * M(B u, z, k t)]\right\} M(z, z, t)} \\
& {[M(z, B u, k t)]^{2} * M(z, B u, k t) \geq\left\{k_{1}[M(B u, z, 2 k t)]+k_{2}[M(B u, z, k t)]\right\}} \\
& {[M(z, B u, k t)]\{[M(z, B u, k t)] * M(z, B u, k t)\} \geq\left\{k_{1}[M(B u, z, 2 k t)]+k_{2}[M(B u, z, k t)]\right\}} \\
& {[M(z, B u, k t)]\{[M(z, B u, k t)]\} \geq\left\{k+k_{2}\right\}[M(B u, z, k t)]} \\
& {[M(z, B u, k t)]^{2} \geq\left\{k+k_{2}\right\}[M(B u, z, k t)]} \\
& {[M(z, B u, k t)] \geq\left\{k+k_{2}\right\}} \\
& {[M(z, B u, k t)] \geq 1} \\
& \therefore B u=z
\end{aligned}
$$

Again since the pair $(\mathrm{B}, \mathrm{T})$ is weakly compatible and $\mathrm{Bu}=\mathrm{Tu}=\mathrm{Z}$ implies $\mathrm{BTu}=\mathrm{TBu}$ or $\mathrm{Bz}=\mathrm{Tz}$. 
To prove $A z=z$ put $x=z, y=x_{2 n+1}$

$$
\begin{aligned}
& {\left[M\left(A z, B x_{2 n+1}, k t\right)\right]^{2} * M\left(A z, B x_{2 n+1}, k t\right) M\left(T x_{2 n+1}, S z, k t\right) \geq\left\{k_{1}\left[M\left(B x_{2 n+1}, S z, 2 k t\right) * M\left(A z, T, x_{2 n+1} 2 k t\right)\right]\right.} \\
& \left.+k_{2}\left[M(A z, S z, k t) * M\left(B x_{2 n+1}, T x_{2 n+1}, k t\right)\right]\right\} M\left(T x_{2 n+1}, S z, t\right) \\
& {[M(A z, z, k t)]^{2} * M(A z, z, k t) M(z, A z, k t) \geq\left\{k_{1}[M(z, A z, 2 k t) * M(A z, z, 2 k t)]\right.} \\
& \left.+k_{2}[M(A z, A z, k t) * M(z, z, k t)]\right\} M(z, A z, t) \\
& {[M(A z, z, k t)] \geq \frac{k_{2}}{1-k_{1}}} \\
& {[M(A z, z, k t)] \geq 1} \\
& \therefore A z=z
\end{aligned}
$$

To prove $\mathrm{Bz}=\mathrm{z}$ Put $\mathrm{x}=\mathrm{z}, \mathrm{y}=\mathrm{z}$

$$
\begin{aligned}
& {[M(A z, B z, k t)]^{2} * M(A z, B z, k t) M(T z, S z, k t) \geq\left\{k_{1}[M(B z, S z, 2 k t) * M(A z, T z, 2 k t)]\right.} \\
& \left.+k_{2}[M(A z, S z, k t) * M(B z, T z, k t)]\right\} M(T z, S z, t) \\
& {[M(z, B z, k t)]^{2} * M(z, B z, k t) M(B z, z, k t) \geq\left\{k_{1}[M(B z, z, 2 k t) * M(z, B z, 2 k t)]\right.} \\
& \left.+k_{2}[M(z, z, k t) * M(B z, B z, k t)]\right\} M(B z, z, t) \\
& {[M(z, B z, k t)]^{2} *[M(z, B z, k t)]^{2} \geq\left\{k_{1}[M(B z, z, 2 k t)]+k_{2}[1]\right\} M(B z, z, t)} \\
& \left\{[M(z, B z, k t)]^{2}\right\} \geq\left\{k_{1}[M(B z, z, 2 k t)]+k_{2}\right\} M(B z, z, k t) \\
& \{[M(z, B z, k t)]\} \geq\left\{k_{1}[M(B z, z, k t)]+k_{2}\right\} \\
& {[M(z, B z, k t)]\left\{1-k_{1}\right\} \geq\left\{k_{2}\right\}} \\
& {[M(z, B z, k t)] \geq \frac{k_{2}}{1-k_{1}}} \\
& {[M(z, B z, k t)] \geq 1} \\
& \therefore B z=z
\end{aligned}
$$

Since $\mathrm{Az}=\mathrm{Bz}=\mathrm{Sz}=\mathrm{Tz}=\mathrm{z}$, we get $\mathrm{z}$ is a common fixed point of $\mathrm{A}, \mathrm{B}, \mathrm{S}$ and $\mathrm{T}$. The uniqueness of the fixed point can be easily proved.

(2.2.5) Example: Let $\mathrm{X}=[0,1], \quad M(x, y, t)=\frac{t}{t+d(x, y)} \quad$ where $\mathrm{d}(\mathrm{x}, \mathrm{y})=|x-y|$

$$
A x=B x=\left\{\begin{array}{l}
\frac{1}{8} \text { if } 0 \leq x \leq \frac{1}{8} \\
\frac{1}{6} \quad \text { if } \frac{1}{8}<x \leq 1
\end{array} \quad S x=T x= \begin{cases}\frac{1}{4}-x \quad \text { if } 0 \leq x \leq \frac{1}{8} \\
\frac{1}{4} \quad \text { if } \frac{1}{8}<x \leq 1\end{cases}\right.
$$

Then $\mathrm{A}(\mathrm{X})=\mathrm{B}(\mathrm{X})=\left\{\frac{1}{8}, \frac{1}{6}\right\}$ while $\mathrm{S}(\mathrm{X})=\mathrm{T}(\mathrm{X})=\left\{\frac{1}{4} \cup\left[\frac{1}{4}, \frac{1}{8}\right]\right\}$ so that the conditions $\mathrm{A}(\mathrm{X})$ $\subseteq \mathrm{T}(\mathrm{X})$ and $\mathrm{B}(\mathrm{X}) \subseteq \mathrm{S}(\mathrm{X})$ are satisfied 
From the example given above, clearly the pairs $(\mathrm{A}, \mathrm{S})$ and $(\mathrm{B}, \mathrm{T})$ are weakly compatible as they commute at coincident point $1 / 8$. But the pairs $(\mathrm{A}, \mathrm{S})$ and $(\mathrm{B}, \mathrm{T})$ are not compatible For this, take a sequence $\mathrm{x}_{\mathrm{n}}=\left(\frac{1}{8}-\frac{1}{n}\right)$ for $\mathrm{n} \geq 1$, then $\lim _{\mathrm{n} \rightarrow \infty} \mathrm{Ax}_{\mathrm{n}}=\lim _{\mathrm{n} \rightarrow \infty} \mathrm{Sx}_{\mathrm{n}}=1 / 8$ and $\lim _{n \rightarrow \infty} A S x_{n}=1 / 6$ also $\lim _{n \rightarrow \infty} S A x_{n}=1 / 8$ So that $\lim _{n \rightarrow \infty} M\left(A S x_{n}, S A x_{n}, t\right) \neq 1$. Also note that none of the mappings are continuous and the rational inequality holds for the values of $0 \leq k_{1}+k_{2} \geq 1$, where $k_{1}, k_{2} \geq 0$. Clearly $1 / 8$ is the unique common fixed point of $\mathrm{A}, \mathrm{B}, \mathrm{S}$ and T.

\section{REFERENCES}

[1] Zadeh L A, Fuzzy Sets,Information and Control,8(1965),338-353.

[2] Balasubramaniam P, Murali Sankar S \& Pant R P, Common fixed point of four mappings in fuzzy metric space, J.Fuzzy Math. 10(2) (2002), 379-384.

[3] George A \& Veeramani P, On some results in fuzzy metric spaces, Fuzzy Sets and Systems, 64 (1994), 395-399.

[4] Grabiec M, Fixed points in fuzzy metric spaces, Fuzzy Sets and Systems 27, (1988), 385-389.

[5] Sharma S, Common fixed point theorems in fuzzy metric spaces, Fuzzy Sets and Systems 127(2002), 345-352.

[6] Cho Y J, Pathak H K, Kang S M \& Jung J S, Common fixed point of compatible maps of type $\beta$ on fuzzy metric spaces, Fuzzy sets and Systems, 93(1998), 99-111.

[7] Jungck G, Compatible mappings and common fixed points (2) Internet. .J.Math \& Math. Sci. (1988), 285-288.

[8] Sisodia K S, Rathore M S, \& Deepak Singh, A common fixed point theorem in fuzzy metric spaces, Vol.5, 2011, No.17, 819-826.

[9] Kutukcu S, Sharma S \& Tokgoz H A, A fixed point theorem in fuzzy metric spaces, Int. J.Math. Analysis, 1(18) (2007), 861-872.

[10] Mishra S N, Sharma N \& Singh S L, Common fixed points of mappings on fuzzy metric spaces, Internet .J.Math \&Math .Sci,17(1994), 253-258.

[11] Pant R P \& Jha K, A Remark on common fixed points of four mappings in a fuzzy metric space, J.Fuzzy Math.12 (2) (2004),433-437.

[12] Srinivas V, B V B Reddy \& Umamaheshwar Rao R, A focus on common fixed point theorem using weakly compatible mappings ,Mathematical Modelling,Vol.2,

No 3, 2012,60-65.

[13] Koireng M \& Yumnam Rohen, Common fixed point theorems of compatible mappings of type(P) of fuzzy metric spaces, Int. Journal of Math. Analysis, vol.6, No.4, 2012, 181-188. 